\title{
Primary renal NUT carcinoma identified by next- generation sequencing: A case report and literature review
}

\author{
Fei Yang \\ Peking University People's Hospital \\ Danhua Shen ( $\square$ shenpath59@163.com ) \\ Junping Shi \\ OrigiMed
}

\section{Short report}

Keywords: Carcinoma, Diagnosis, Kidney, Sarcoma

Posted Date: February 20th, 2020

DOI: https://doi.org/10.21203/rs.2.24123/v1

License: @ (i) This work is licensed under a Creative Commons Attribution 4.0 International License. Read Full License 


\section{Abstract}

Background NUT carcinoma is a rare aggressive squamous cell carcinoma subtype genetically defined by NUTM1 rearrangements. NUT carcinoma usually has a primitive differentiation state and can be easily misdiagnosed as an undifferentiated carcinoma or Ewing sarcoma.

Case presentation We report a case of NUT carcinoma of renal origin initially diagnosed as a malignant small round-cell tumor, likely to be Ewing sarcoma/primitive neuroectodermal tumor. Based on nextgeneration sequencing (NGS), the diagnosis was revised to NUT carcinoma with a characteristic NUTM1 rearrangement. The patient relapsed after surgery and received a standard NUT carcinoma treatment. However, due to advanced neoplasm progression, first-line chemotherapy failed and the patient died.

Conclusion Routine NUT immunohistochemistry staining, NGS, and/or fluorescent in situ hybridization for poorly differentiated carcinoma and sarcoma tumors can help avoid misdiagnosis of NUT carcinomarelated tumors, allowing patients to benefit from bromodomain and extra-terminal motif inhibitor therapy.

\section{Background}

Genetically-rearranged nuclear protein in testis (NUT) midline carcinoma (NUT carcinoma) is a relatively rare, highly malignant tumor [1]. A characteristic feature of approximately $70 \%$ of NUT carcinomas is the translocation $\mathrm{t}(15 ; 19)(q 14 ; \mathrm{p} 13)$, which leads to the gene fusion of NUT midline carcinoma family member 1 (NUTM1) with bromodomain containing 4 (BRD4). Other partner genes that fuse with NUTM1 include bromodomain containing 3 (BRD3) and nuclear receptor binding SET domain protein 3 (NSD3) [2].

NUT carcinoma generally exhibits primitive differentiation with an abrupt squamous epithelial component. However, nearly $50 \%$ of the cases lack a squamous epithelial component and are often misdiagnosed as undifferentiated carcinoma or Ewing's carcinoma. With the rapid development of molecular pathology, next-generation sequencing (NGS) has emerged as a means to provide unique advantages for the diagnosis of poorly differentiated cancers that lack characteristic histological features. We report a case of primary renal NUT carcinoma that was identified by NGS to have a NUTM1 rearrangement and present a review of the relevant literature.

\section{Case Report}

A 41-year-old male visited a local hospital in September 2018 due to lumbago. His past medical history and family history were unremarkable. An enhanced computed tomography (CT) scan revealed a soft tissue density shadow $7.1 \mathrm{~cm} \times 2.6 \mathrm{~cm}$ in the left kidney. The density was uneven with a local protrusion from the renal silhouette. Upon contrast enhancement of the CT scan, the density intensified. Laparoscopic radical excision surgery was performed. Based on gross examination, the resected kidney specimen was $14.0 \mathrm{~cm} \times 10.0 \mathrm{~cm} \times 6.5 \mathrm{~cm}$ with a mass at the inferior pole being $7.0 \mathrm{~cm} \times 5.7 \mathrm{~cm}$. The surface of the specimen was gray-white and gray-red of medium hardness with an unclear boundary. The mucosae of the renal pelvis and ureter were smooth. Microscopic examination revealed a diffuse, patchy 
infiltration of tumor cells. The cells were round or oval shape and ranged in size from small to medium. The cytoplasm was relatively rich and eosinophilic with plasmacytoid and epithelioid morphologies. Nuclear atypia was significant and some nuclei were vesicula-like. Nucleoli and karyokinesis were obvious. Focal patchy necrosis was present with perineural invasion in some foci. There was extensive vascularization of the tumor stroma (Fig. 1A).

Immunohistochemistry demonstrated positive expression of cytokeratin( CK )(Fig. 1B), CD99, p63 (Fig. 1C), and FLI-1. Staining was negative for the expression of desmin, S-100, BCOR, and other lymphoid markers. The Ki-67 proliferation index in the region of interest reached $25 \%$. Based on postoperative pathology results, a diagnosis was made of a malignant small round-cell tumor with a high likelihood of Ewing sarcoma/primitive neuroectodermal tumor (PNET). However, fluorescent in situ hybridization (FISH) was negative as the Ewing sarcoma breakpoint region 1 (EWSR1) could not be detected. Therefore, a further diagnosis was needed. After obtaining consent from the patient, the surgically resected tissues and peripheral blood were tested for YuanSu $\mathrm{S}^{\mathrm{TM}} 450$ tumor-related genes at both the DNA and RNA level. The testing was carried out by OrigiMed (Shanghai, China), which is a College of American Pathologists (CAP) accredited and Clinical Laboratory Improvement Amendments (CLIA) certified laboratory. NGS revealed the tumor harbored only one reported mutation at the DNA and RNA level, a BRD4 exon 14-NUTM1 exon3 fusion (Fig. 2). The tumor mutational burden (TMB) was 0.8 mutations/megabase and the microsatellite status was stable. Further immunohistochemistry analysis demonstrated positive expression of NUT protein (Fig. 1D). Based on these results, a diagnosis of primary renal NUT carcinoma was made.

During the 5-month post-operative follow up, imaging showed enlarged perirenal lymph nodes and multiple nodules in both lungs. Although there was a high probability of metastasis, aspiration biopsy was not performed due to the position of lung lesions. Based on the standard treatment for NUT carcinoma, a 4-week course of vincristine, doxorubicin, cyclophosphamide/ifosfamide, etoposide (VDC/IE) chemotherapy was initiated. Three months later, the patient still presented extensive peritoneal dissemination. The patient ultimately died of disease progression with the overall survival from the time of diagnosis being eight months.

\section{Discussion}

NUT carcinoma is a rare, highly malignant tumor of unclear origin that is often considered a poorly differentiated squamous cell carcinoma; however, its clinicopathological features are different from those of classical squamous cell carcinoma. NUT carcinomas can occur at any age (0-81.7 years), but primarily affects children, adolescents, and young adults. It typically develops at the midline with $50 \%$ of cases occurring in the aerodigestive tract and $41 \%$ occurring in the mediastinum [3]. However, it can also occur in parenchymal organs, including the urinary bladder, mammary gland, endometrium, kidney, and orbit, and is able to invade soft tissues and bone.

During the initial hospital visit by the patient described in the current case report, the tumor was limited to the kidney and imaging did not show significant abnormalities at any other sites. Accordingly, the kidney 
was considered to be the primary site. Primary renal NUT carcinoma is extremely rare. Our literature search identified reports of only five other cases of primary renal NUT carcinoma [4-7]. The clinicopathological features of the current patient and those of the previously reported five cases are summarized in Table 1.

Typical NUT carcinoma morphology is a patchy infiltration of small-to-medium-sized undifferentiated tumor components, low-to-moderate tumor cell cytoplasm volume, and active karyokinesis. Sudden triggering of squamous epithelial cell differentiation may occur in approximately half of the cases. In contrast to typical squamous cell carcinoma, which is characterized by rich polymorphism, the morphology of NUT carcinoma cells is relatively consistent; however, NUT carcinoma is not typically found in situ [8]. The current case of NUT carcinoma was similar to the five other reported cases in that cell differentiation was relatively primitive, all cells lacked squamous epithelial characteristics, and tumors demonstrated diffuse but strongly positive expression of CD99. FISH was negative in our patient for an EWSR1 fusion. EWSR1 was reportedly fused with FLI1 in only 85\% of cases [9]; Although the FISH results indicated that EWSR1 escaped probe detection of gene breakage and translocation, this negative result could not completely rule out a diagnosis of Ewing sarcoma. Therefore, a diagnosis of NUT carcinoma was made only after NGS analysis.

A review of the literature revealed that NUT carcinoma is often misdiagnosed as Ewing sarcoma/PNET. A characteristic feature of NUT carcinoma based on immunohistochemistry is that $>50 \%$ of tumor nuclei are positive for NUTM1, with a sensitivity and specificity of $87 \%$ and $100 \%$, respectively. By combining immunohistochemistry with FISH, the diagnostic sensitivity can reach $100 \%$ [10]. While NUT immunohistochemistry can be used as a primary screen, a definitive diagnosis of NUT carcinoma requires the molecular detection of NUTM1 rearrangement/fusion. NUTM1 has multiple fusion partners. Two-thirds of the patients have fusions between NUTM1 and BRD4, while the remaining exhibit fusions between NUTM1 and BRD3 or NSD3 [11]. Although the partner gene of NUTM1 of both the five primary renal NUT carcinomas from the literature and our current case all shows BRD4, whether the partner gene is related to the primary site needs further investigation.

The rapidly increasing use of NGS has led to the discovery of additional partner genes for NUTM1, including CIC, BCORL1, MYXD1, and MGA [6 12 13]. This has also widened the range of tumor histology targets. A report by den Bakker et al.,[14] described a parotid tumor as the primary site, which involved a BRD4-MUTM1 fusion. A cartilage-differentiated sarcoma component was also observed. Furthermore, of 26 cases of tumors with NUTM1 rearrangements reported by Stevens et al. [14], one was a primary pulmonary tumor with an MGA-NUTM1 fusion, which presented a myxoid chondrosarcoma morphology [13]. Therefore, tumors with NUTM1 rearrangements are not limited to NUT carcinomas, but may also be present in NUT sarcomas. The relationship between NUT sarcomas and the more common NUT carcinomas requires further investigation with some investigators suggesting that this type of tumor should be generically classified as a "NUT-related tumor" [5]. 
The clinical course of NUT carcinoma is naturally risk-prone and its prognosis is extremely poor. At the time of initial diagnosis, most patients already have distant metastases. Moreover, the disease responds poorly to traditional radiotherapy and chemotherapy with the median survival being only 6.7 months [15]. In our patient, even after surgery and a post-operative 4-wk course of VDC/IE chemotherapy, the disease was not controlled. Metastasis to both lungs and the peritoneum had occurred, as well as extensive peritoneal spreading. The patient died eight months after surgery.

In recent years, the emergence of oral bromodomain and extra-terminal domain (BET) inhibitors targeting the BRD2, BRD3, and BRD4 proteins has been beneficial to the management of NUT carcinoma. BET inhibitors mimic the structure of acetylated histone lysine residues and thereby interrupt the interaction between BRD4 and actual acetylated histone lysine. This results in the attenuation of abnormal transcription and induces tumor cell differentiation. However, oral BET inhibitors may not be effective for patients without BRD-NUTM1 fusions [16].

\section{Conclusion}

NGS not only is beneficial in the diagnosis of NUT carcinoma, but also allows for the use of more targeted therapies as a result of being able to assess the precise mutations of cancer-related genes. Our experience with the patient described in this report has led us to performing NUT immunohistochemistry on all poorly differentiated tumors, both carcinomas and sarcomas. This can then be followed by NGS and FISH for validation in order to avoid missing or incorrectly diagnosing NUTM1 fusion-related tumors, thereby benefitting more patients.

\section{Abbreviations}

NGS Next-generation sequencing

NUT midline carcinoma family member 1

BRD4 bromodomain containing 4

BRD3 bromodomain containing 3

NSD3 nuclear receptor binding SET domain protein 3

CT computed tomography

CK cytokeratin

PNET primitive neuroectodermal tumor

FISH fluorescent in situ hybridization

EWSR1 Ewing sarcoma breakpoint region 1 
CAP College of American Pathologists

CLIA Clinical Laboratory Improvement Amendments

TMB tumor mutational burden

\section{Declarations}

\section{Acknowledgements}

We owe thanks to the patient and his family. We thank OrigiMed for NGS technical support and scientific comments.

\section{Authors' contributions}

Conception/Design: Danhua Shen

Provision of study material or patients: Danhua Shen

Manuscript writing: Fei Yang, Junping Shi

Final approval of manuscript: Danhua Shen, Fei Yang ,Junping Shi

All authors have read and approved the submitted version of the manuscript.

\section{Funding}

No funding

\section{Availability of data and materials}

The dataset supporting the conclusion of this article is included within the article.

\section{Ethics approval and consent to participate}

Not applicable

\section{Consent for publication}

Written informed consent was obtained from the patient for the publication of this case report and any accompanying images. A copy of the consent form is available for review by the Editor of Diagnostic Pathology

\section{Competing interests}

The authors declare that they have no competing interests.

\section{Contributor Information}


Danhua Shen, Email: shenpath59@163.com

Fei Yang, Email:18800167092@163.com

Junping Shi, Email:fish0219916@126.com

\section{References}

1. French CA, Kutok JL, Faquin WC, et al. Midline carcinoma of children and young adults with NUT rearrangement. Journal of clinical oncology : official journal of the American Society of Clinical Oncology 2004;22(20):4135-9 doi: 10.1200/JC0.2004.02.107[published Online First: Epub Date]|.

2. Kubonishi I, Takehara N, Iwata J, et al. Novel t(15;19)(q15;p13) chromosome abnormality in a thymic carcinoma. Cancer research 1991;51(12):3327-8

3. Bishop JA, French CA, Ali SZ. Cytopathologic features of NUT midline carcinoma: A series of 26 specimens from 13 patients. Cancer cytopathology 2016;124(12):901-08 doi: 10.1002/cncy.21761 [published Online First: Epub Date]|.

4. Sirohi D, Garg K, Simko JP, Grenert JP. Renal NUT carcinoma: a case report. Histopathology 2018;72(3):528-30 doi: 10.1111/his.13368[published Online First: Epub Date]|.

5. Dickson BC, Sung YS, Rosenblum MK, et al. NUTM1 Gene Fusions Characterize a Subset of Undifferentiated Soft Tissue and Visceral Tumors. The American journal of surgical pathology 2018;42(5):636-45 doi: 10.1097/PAS.0000000000001021[published Online First: Epub Date]|.

6. Zhu Y, Liu YF, Zhao J, Yu YW. [BRD4-NUT fusion oncogene carcinoma in the kidney]. Zhonghua bing li xue za zhi = Chinese journal of pathology 2019;48(3):237-39 doi: 10.3760/cma.j.issn.05295807.2019.03.013[published Online First: Epub Date]|.

7. French CA. Demystified molecular pathology of NUT midline carcinomas. Journal of clinical pathology 2010;63(6):492-6 doi: 10.1136/jcp.2007.052902[published Online First: Epub Date]|.

8. French CA. NUT Carcinoma: Clinicopathologic features, pathogenesis, and treatment. Pathology international 2018;68(11):583-95 doi: 10.1111/pin.12727[published Online First: Epub Date]|.

9. Balamuth NJ, Womer RB. Ewing's sarcoma. The Lancet. Oncology 2010;11(2):184-92 doi: 10.1016/S1470-2045(09)70286-4[published Online First: Epub Date].

10. Haack H, Johnson LA, Fry CJ, et al. Diagnosis of NUT midline carcinoma using a NUT-specific monoclonal antibody. The American journal of surgical pathology 2009;33(7):984-91 doi: 10.1097/PAS.0b013e318198d666[published Online First: Epub Date]|.

11. Alekseyenko AA, Walsh EM, Zee BM, et al. Ectopic protein interactions within BRD4-chromatin complexes drive oncogenic megadomain formation in NUT midline carcinoma. Proceedings of the National Academy of Sciences of the United States of America 2017;114(21):E4184-E92 doi: 10.1073/pnas.1702086114[published Online First: Epub Date]|.

12. Schaefer IM, Dal Cin P, Landry LM, Fletcher CDM, Hanna GJ, French CA. CIC-NUTM1 fusion: A case which expands the spectrum of NUT-rearranged epithelioid malignancies. Genes, chromosomes \& 
cancer 2018;57(9):446-51 doi: 10.1002/gcc.3[published Online First: Epub Date]|.

13. Stevens TM, Morlote D, Xiu J, et al. NUTM1-rearranged neoplasia: a multi-institution experience yields novel fusion partners and expands the histologic spectrum. Modern Pathology 2019;32(6):764-73 doi: 10.1038/s41379-019-0206-z[published Online First: Epub Date]|.

14. den Bakker MA, Beverloo BH, van den Heuvel-Eibrink MM, et al. NUT midline carcinoma of the parotid gland with mesenchymal differentiation. The American journal of surgical pathology 2009;33(8):1253-8 doi: 10.1097/PAS.0b013e3181abe120[published Online First: Epub Date]|.

15. Bauer DE, Mitchell CM, Strait KM, et al. Clinicopathologic features and long-term outcomes of NUT midline carcinoma. Clinical cancer research : an official journal of the American Association for Cancer Research 2012;18(20):5773-9 doi: 10.1158/1078-0432.CCR-12-1153[published Online First: Epub Date]|.

16. Seim NB, Philips RHW, Schoenfield L, et al. NUT Midline Carcinoma of the Sublingual Gland: Clinical Presentation and Review. Head and neck pathology 2017;11(4):460-68 doi: 10.1007/s12105-0170809-y[published Online First: Epub Date]|.

\section{Figures}



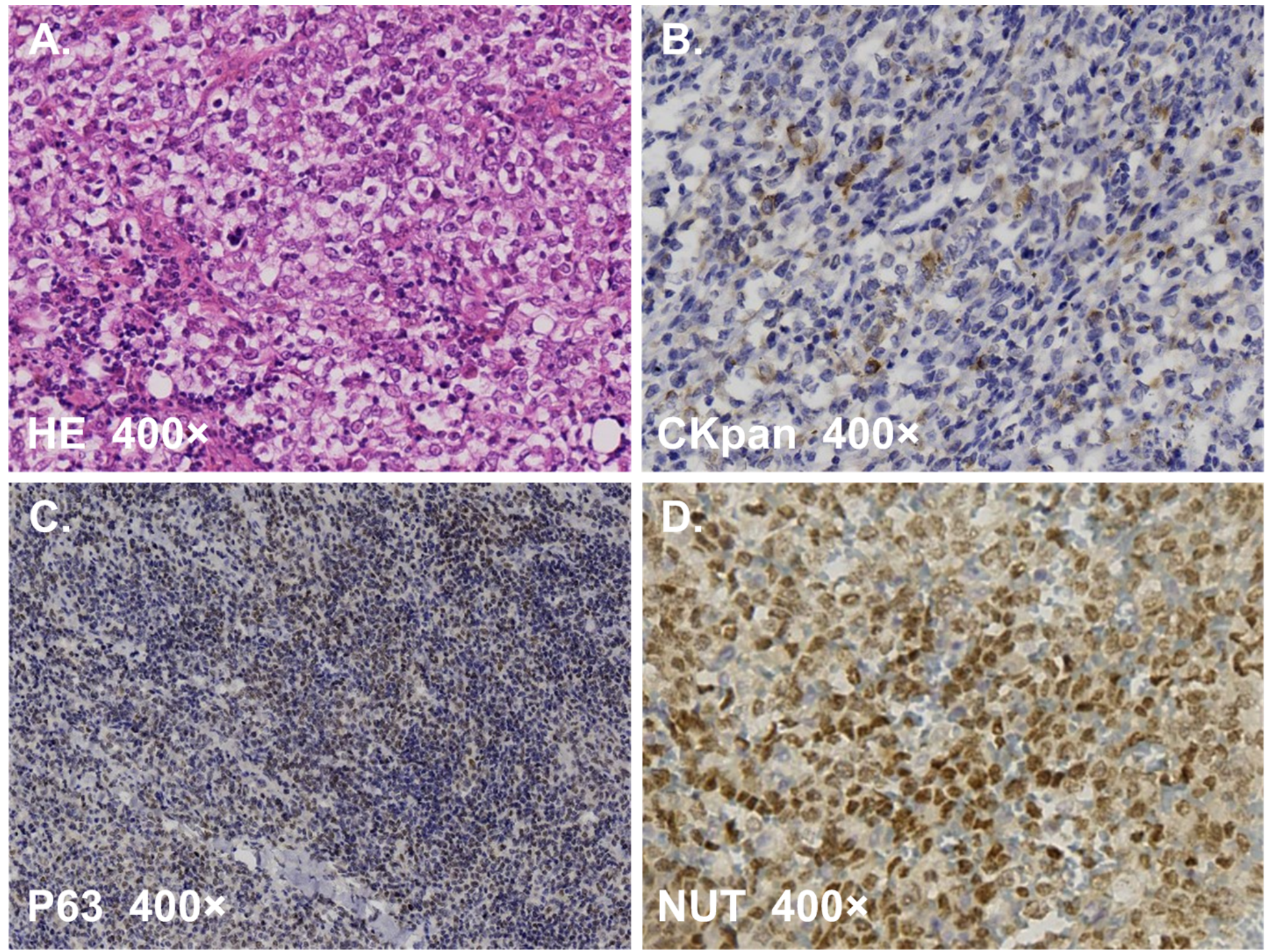

\section{Figure 1}

Extensive vascularization of the tumor stroma (A). Immunohistochemistry demonstrated positive expression of cytokeratin( CK )(B), CD99, p63 (C), (D)ositive expression of NUT protein 
A.

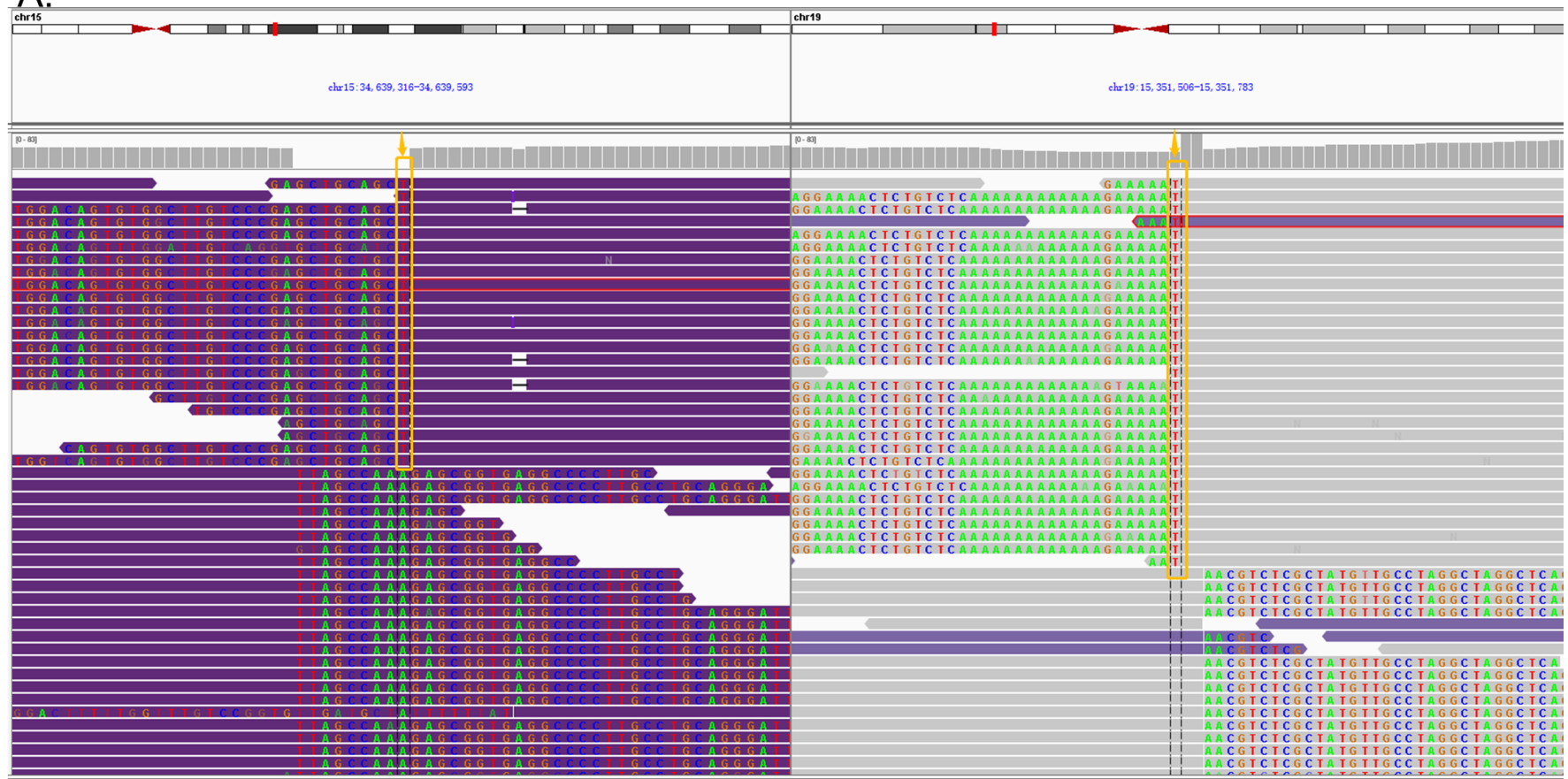

CCTAGGCAACATAGCGAGACGTCATCTCTACAATTTTTCTTTTTTTTTTTTTGAGACAGACATCCCTGCAGGCAAGGGGCCTCACCGCTCTTTGGCTAGCTGCAGCTCGGGACCAAGCCACACTGTCCACA
NUTM1
BRD4

B.
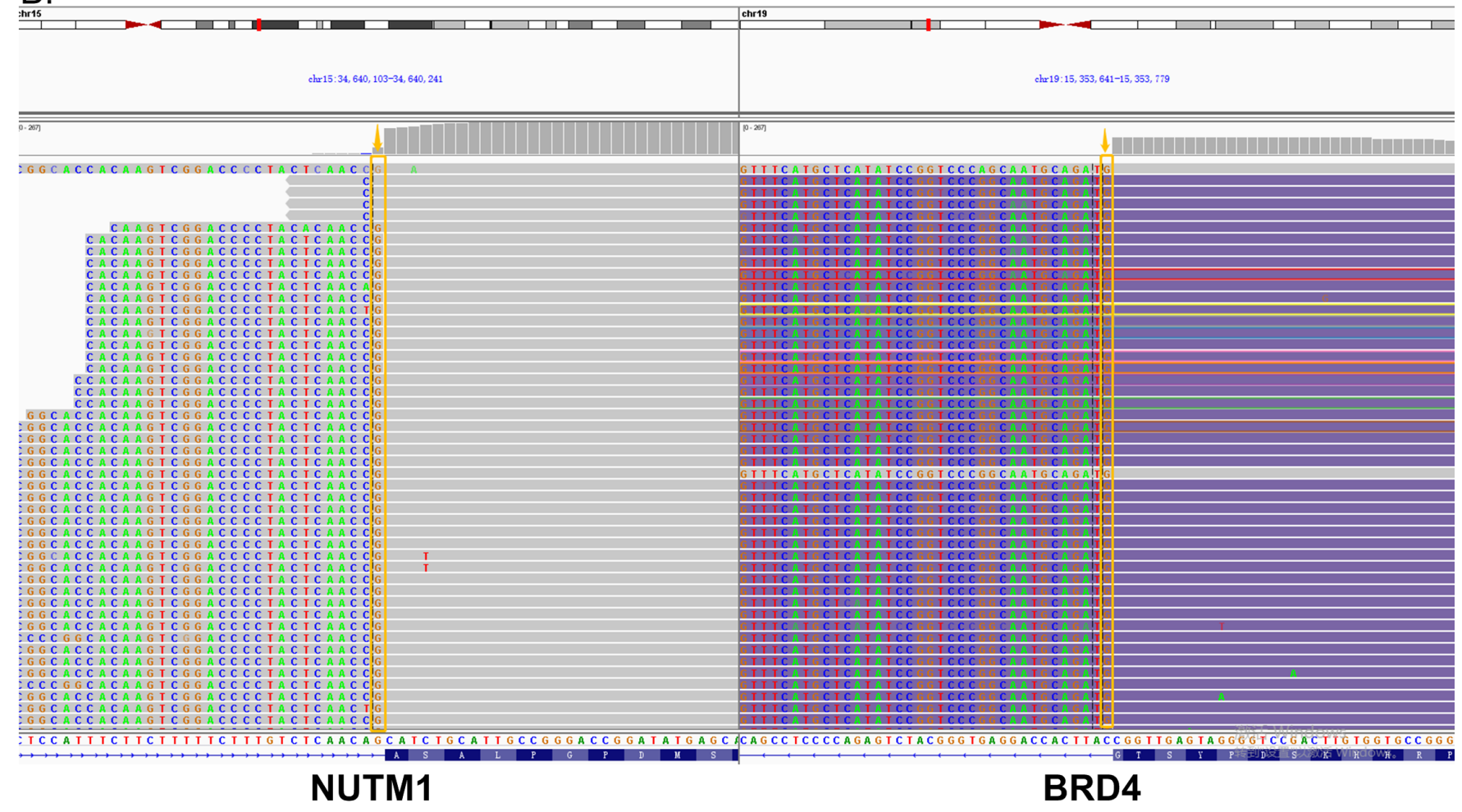

Figure 2

The tumor harbored only one reported mutation at the DNA and RNA level, a BRD4 exon 14-NUTM1 exon3 fusion 